\section{Reply: The Importance of an Adequate Surgical Template During Salvage Lymph Node Dissection for Node-Recurrent Prostate Cancer}

\begin{abstract}
REPLY: In line with the comments by Bravi et al. on our article (1), prostate-specific membrane antigen (PSMA) PET demonstrated low sensitivity but high specificity and negative predictive value for the detection of pelvic nodal metastases, compared with histopathology, in a multicenter prospective phase III imaging study using blinded independent central reads (2). PSMA PET detection inversely correlates with the size of tumor deposits, and thus PET is prone to missing micrometastatic disease (3). Despite underestimation on a single-lesion level (4), PSMA PET positivity raises a red flag for diseased regions (1). Because nodal spread follows lymph drainage anatomy, PSMA PET guidance toward regions at risk seems feasible. The ongoing prospective ProsTone trial (NCT04271579) will assess whether unilateral pelvic lymph node dissection on the PSMA PET-positive side will lead to an improved trade-off between efficacy and toxicity by sparing potentially nondiseased contralateral nodal regions. We agree with Bravi and colleagues that suggesting the adoption of an adequate template will be key to maximizing the benefit for patients undergoing local salvage therapy. In addition, standardized reporting of PSMA PET using a rational anatomic framework together with implementation into clinical trials on local therapy will be key to defining the future role of PSMA PET in treatment guidance (5).
\end{abstract}

\section{REFERENCES}

1. Farolfi A, Ilhan H, Gafita A, et al. Mapping prostate cancer lesions before and after unsuccessful salvage lymph node dissection using repeat PSMA PET. J Nucl Med. 2020;61:1037-1042.

2. Hope TA, Armstrong WR, Murthy V, et al. Accuracy of ${ }^{68} \mathrm{Ga}$-PSMA-11 for pelvic nodal metastasis detection prior to radical prostatectomy and pelvic lymph node dissection: a multicenter prospective phase III imaging study [abstract]. J Clin Oncol. 2020; $38(15$, suppl $) 5502$

3. Jilg CA, Drendel V, Rischke HC, et al. Detection rate of ${ }^{18} \mathrm{~F}$-choline PET/CT and ${ }^{68} \mathrm{Ga}-$ PSMA-HBED-CC PET/CT for prostate cancer lymph node metastases with direct link from PET to histopathology: dependence on the size of tumor deposits in lymph nodes. J Nucl Med. 2019;60:971-977.

4. Horn T, Krönke M, Rauscher I, et al. Single lesion on prostate-specific membrane antigen-ligand positron emission tomography and low prostate-specific antigen are prognostic factors for a favorable biochemical response to prostate-specific membrane antigen-targeted radioguided surgery in recurrent prostate cancer. Eur Urol. 2019;76: 517-523.

5. Eiber M, Herrmann K, Calais J, et al. Prostate Cancer Molecular Imaging Standardized Evaluation (PROMISE): proposed miTNM classification for the interpretation of PSMA-ligand PET/CT. J Nucl Med. 2018;59:469-478.

\section{Wolfgang Peter Fendler Andrea Farolfi \\ ${ }^{\star}$ University Hospital Essen Essen, Germany \\ E-mail: wolfgang.fendler@uk-essen.de}

Published online Apr. 16, 2021.

DOI: 10.2967/jnumed.121.262271

(C) 2021 by the Society of Nuclear Medicine and Molecular Imaging.

\section{V/Q SPECT/CT in the Time of COVID-19: Changing the Order to Improve Safety Without Sacrificing Accuracy}

TO THE EDITOR: Various nuclear medicine associations and colleagues (1-6) have discussed whether a ventilation examination should be included at all when performing ventilation-perfusion (V/Q) scans for diagnosis of pulmonary embolism during the severe acute respiratory syndrome coronavirus 2 (SARS-CoV-2) pandemic. This consideration was prompted by the concern that a ventilation scan may be an aerosol-prone maneuver and, thus, carry a potential risk of infecting the personnel. Usually, the V/Q scan procedure starts with a ventilation scan, which is followed by a perfusion scan and is eventually closed with a low-dose CT scan, if available. This sequence is traditionally chosen for a V/Q scan because it is easier to surpass the ventilation activity with the perfusion marker than vice versa. Instead of completely eliminating the ventilation scan, resulting in a reduction in specificity (7) and all its negative consequences - such as bleeding events due to unnecessary anticoagulation-we suggest modification of the workflow during the pandemic such that the scan routinely starts with perfusion SPECT (with a somewhat lower administered activity than usual, associated with an increased acquisition time) and proceeds with low-dose CT, if applicable. Ventilation SPECT (with a somewhat higher administered activity than usual, associated with a decreased acquisition time) would be performed only when there are perfusion deficits that are not sufficiently explained by structural findings on low-dose CT. If the patient is SARS-CoV-2-positive or there are COVIDlike findings on low-dose CT, it would remain at the discretion of the physician whether a ventilation scan is performed, under appropriate security measures. By doing so, we can reduce the number of ventilation scans and avoid the aforementioned discussion by various nuclear medicine associations and colleagues $(1-6)$.

Figure 1 shows a representative example of the proposed approach in a recently bedridden 80 -y-old woman with dyspnea, thoracic pain, and an increased $D$-dimer level who was referred from an external hospital to rule out pulmonary embolism. We started by injecting about $45 \mathrm{MBq}$ of ${ }^{99 \mathrm{~m}} \mathrm{Tc}$-macroaggregated albumin and performing perfusion SPECT/CT. Since we found relevant perfusion deficits and unremarkable findings on low-dose CT, we subsequently also performed ventilation SPECT with about $88 \mathrm{MBq}$ of ${ }^{99 \mathrm{~m}}$ Tc-Technegas (Cyclomedica) (net ventilated activity calculated from the projection data). The ventilation and perfusion studies showed mismatched findings typical of pulmonary embolism (Fig. 1A). In combination with the normal results from the low-dose CT examination (Fig. 1B), these findings allowed us to detect pulmonary embolism with the highest degree of certainty, according to Gutte et al. (7).

Overall, we believe that the suggested routine reversal of the traditional workflow helps to minimize aerosol-prone and potentially infectious maneuvers without compromising the accuracy of pulmonary embolism diagnostics.

(C) 2021 by the Society of Nuclear Medicine and Molecular Imaging 